\title{
Publisher Correction: Local management actions can increase coral resilience to thermally-induced bleaching
}

\author{
Elizabeth C. Shaver (D), Deron E. Burkepile (D) and Brian R. Silliman
}

Correction to: Nature Ecology \& Evolution https://doi.org/10.1038/s41559-018-0589-0, published online 18 June 2018.

In the version of this Brief Communication originally published, the two instances of 'natural-to-high' in the sixth and seventh paragraphs were incorrect; they should have read 'naturally high'.

In the final sentence of the sixth paragraph, ' $\sim 100 \mathrm{~m}$ ' was incorrect; it should have read ' $\sim 100 \mathrm{~m}^{2}$ '.

On the $x$-axis Fig. 1d, the first bar was incorrectly labelled 'None'; it should have read 'Removal'. Likewise, within Fig. 1d, 'High versus none' and 'Average versus none' were incorrect; they should have read 'High versus removal' and 'Average versus removal'.

Owing to a technical error, some text was mistakenly included in the second sentence of the paragraph beginning 'Sea surface temperature': the phrase 'bleachinexcluded due to statistically lower snail densitiesg severity' should have just read 'bleaching severity'.

These issues have now been corrected. 